Check for updates

Cite this: RSC Adv., 2017, 7, 46480

Received 10th August 2017

Accepted 15th September 2017

DOI: $10.1039 / \mathrm{c} 7 \mathrm{ra0} 8845 \mathrm{k}$

rsc.li/rsc-advances

\section{Preparation of polyurethane/polyvinyl alcohol hydrogel and its performance enhancement via compositing with silver particles $\uparrow$}

\author{
Xiuqi Li, ${ }^{\text {ab }}$ Yanjiao Jiang, ${ }^{\text {bc }}$ Fu Wang, ${ }^{\text {b } Z e n g j i e ~ F a n, ~}{ }^{\mathrm{c}}$ Haining Wang, ${ }^{\mathrm{b}}$ Caihong Tao*a \\ and Zhaofeng Wang $\mathbb{D i D}^{* \mathrm{~b}}$
}

\begin{abstract}
In this study, polyurethane/polyvinyl alcohol hydrogel was prepared by chemical cross-linking. Silver particles were further composited in the hydrogel via an in situ synthesis technique. The chemical structure and porous morphology were determined by Fourier transform infrared spectroscopy, X-ray photoelectron spectroscopy and field-emission scanning electron microscopy. The swelling abilities, mechanical behaviors, tribological properties, biocompatibilities and antibacterial properties of the hydrogels were investigated. The results suggest that the incorporation of silver particles in polyurethane/polyvinyl alcohol hydrogels could enhance the Young's modulus, tensile strength and elongation, as well as reduce the friction coefficient, while maintaining hydrogels with a moderate water absorption ability and good biocompatibility. Moreover, the addition of silver particles could endow the polyurethane/polyvinyl alcohol hydrogel with prominent antibacterial performance, suggesting that polyurethane/polyvinyl alcohol/silver composite hydrogels could be applied as dressings for wound healing.
\end{abstract}

\section{Introduction}

As the first defense line of the human body, the skin can protect human tissues and organs by preventing invasions from external pathogenic microbial, physical and chemical activities. ${ }^{1,2}$ When a large area of skin is injured, wound exposure results in bacterial infection, leading to serious complications. ${ }^{3}$ Therefore, it is required to cover wound dressings to promote wound healing. At present, the medical dressings, such as dry gauze and oil gauze, employed in clinics just play a passive protective role based on the barrier effect. ${ }^{4}$ However, in order to efficiently promote wound healing, there is an urgent need to develop some novel dressing materials with an active healing function. For this purpose, a variety of polymeric dressing materials such as foams, films, hydrocolloids, alginates, and hydrogels have been currently verified to promote wound healing effectively. ${ }^{5}$

Hydrogel, as a wet and soft material, has received wide attention in tissue engineering, ${ }^{6}$ drug delivery carriers, ${ }^{7}$ actuators, ${ }^{8}$ sensors ${ }^{9}$ and bio-imaging, ${ }^{\mathbf{1 0}}$ and even optoelectronic

${ }^{a}$ School of Chemical and Biological Engineering, Lanzhou Jiaotong University, Lanzhou, 730070, PR China. E-mail: taoch@mail.lzjtu.cn

${ }^{b}$ State Key Laboratory of Solid Lubrication, Lanzhou Institute of Chemical Physics, Chinese Academy of Sciences, Lanzhou, Gansu 730000, China. E-mail: zhfwang@ licp.cas.cn

${ }^{c}$ School of Stomatology, Lanzhou University, Lanzhou, 730000, China

† Electronic supplementary information (ESI) available: Details of mechanical properties and antibacterial performance. See DOI: 10.1039/c7ra08845k devices. ${ }^{11}$ To date, extensive research has focused on their mechanical weakness that seriously limits the related practical applications, while different structural hydrogels including double-network (DN) hydrogel, ${ }^{12,13}$ topological (TP) hydrogel, ${ }^{14}$ and nanocomposite (NC) hydrogel, ${ }^{\mathbf{1 5}, 16}$ have been synthesized to improve their mechanical properties. In addition, hydrogels have also drawn great attention as effective wound dressings because they are capable of providing a moist environment at the wound-dressing interface, allowing oxygen to diffuse, absorbing excess exudates, and preventing body fluid loss. At the same time, the easy functionalization of hydrogels also opens up the opportunity for loading antibacterial drugs, achieving initiative therapy for wounds. ${ }^{17-19}$

Polyurethane (PU), which has urethane linkages within the backbone, can be prepared by polyaddition polymerization using isocyanate and polyol. Because of their excellent biocompatibility, low biological toxicity, desirable mechanical properties, and high molecular design freedom, various polyurethane-based materials have been investigated for biomedical applications. ${ }^{20-22}$ For example, Zhou et al. prepared $\mathrm{pH}$-sensitive polyurethanes using caprolactone derivatives in the soft segment and L-lysine ethyl ester diisocyanate in the hard segment, which can be potential materials as biodegradable and biocompatible multifunctional carriers for active intracellular drug delivery. ${ }^{23}$ PU-based hydrogels were also employed for applications in wound healing. For example, Yoo et al. prepared polyurethane/polyethylene glycol (PU/PEG) hydrogels by polyaddition reaction in an emulsion system. ${ }^{24}$ The PU/PEG hydrogels exhibited high water absorption and 
water vapor transmission rates, and were confirmed to have better wound healing efficacy of a full thickness rat wound model than that of gauze without any significant adverse reactions.

In this study, the waterborne polyvinyl alcohol (PVA) molecules were designed to crosslink with PU. To further enhance the performance of the PU/PVA hydrogel, silver nanoparticles were introduced by an in situ synthesis technique. The asprepared PU/PVA/Ag nanocomposite hydrogels were confirmed to have a uniform porous morphology, desirable mechanical properties, excellent biocompatibility, and outstanding antibacterial activities against $E$. coli and $S$. aureus. Moreover, the PU/PVA/Ag hydrogels show a relatively low coefficient of friction, in the range of 0.2 to 0.35 , indicating that the hydrogel dressings could be applied in wounds located at various sites of human skin without hampering daily actions.

\section{Experimental}

\section{Materials}

Hexamethylene diisocyanate (HMDI), PVA $\left(M_{\mathrm{w}}=67000 \mathrm{Da}\right)$ and trisodium citrate dehydrate $\left(\mathrm{Na}_{3} \mathrm{C}_{6} \mathrm{H}_{5} \mathrm{O}_{7} \cdot 2 \mathrm{H}_{2} \mathrm{O}\right)$ were purchased from Aladdin (China); polycaprolactone diol (PCL, $\left.M_{\mathrm{w}}=2000 \mathrm{Da}\right)$ was obtained from Sigma-Aldrich. Dimethyl sulfoxide (DMSO, solvent) was purchased from Lianlong Bohua (Tianjin) Pharmaceutical Chemical Co., Ltd. (China). Silver nitrate $\left(\mathrm{AgNO}_{3}\right)$ was purchased from Sinopharm Chemical Reagent Co. Ltd. (China).

\section{Preparation of PU/PVA hydrogels}

PVA and PCL were dried in a vacuum oven at a pressure of 50 mbar and a temperature of $80{ }^{\circ} \mathrm{C}, 24 \mathrm{~h}$ before the commencement of the experiments. Then, $2.5 \mathrm{mmol}$ of PCL was dissolved in $100 \mathrm{~mL}$ of DMSO and poured into a three-necked reactor purged with nitrogen gas $\left(\mathrm{N}_{2}\right)$. The reactor was immersed into an oil bath at $80{ }^{\circ} \mathrm{C}$. After that, $5 \mathrm{mmol}$ of HMDI was dissolved in $8.4 \mathrm{~mL}$ of DMSO, and the HMDI/DMSO solution was added dropwise into the reactor with stirring for $10 \mathrm{~h}$ to prepare urethane prepolymers (PPU). The synthetic route of PPU is presented in Scheme S1 (ESI $\dagger$ ). At the same time, $5.84 \mathrm{~g}$ of PVA powder was dissolved in $48.66 \mathrm{~mL}$ DMSO, and the PVA/DMSO solution was obtained at $90{ }^{\circ} \mathrm{C}$ with stirring. After cooling to room temperature, the PPU solution was poured into PVA and stirred evenly, and then the mixture was poured into the mold for crosslinking at room temperature for $12 \mathrm{~h}$. Then, the samples were immersed in $70 \%$ ethanol solution for $24 \mathrm{~h}$ to remove the residual solvents and unreacted monomers, and PU/ PVA hydrogels were obtained.

\section{Loading of silver particles into PU/PVA hydrogels}

The PU/PVA hydrogels were immersed in distilled water for $24 \mathrm{~h}$ and then transferred to a silver nitrate solution $\left(0.5 \mathrm{mmol} \mathrm{L}^{-1}\right.$, $1 \mathrm{mmol} \mathrm{L}^{-1}, 5 \mathrm{mmol} \mathrm{L}^{-1}, 10 \mathrm{mmol} \mathrm{L}^{-1}$ ) for another $24 \mathrm{~h}$. The silver ion loaded PU/PVA hydrogels were subsequently transferred into a beaker containing $10 \mathrm{mmol} \mathrm{L}^{-1}$ of trisodium citrate solution, allowing for $24 \mathrm{~h}$ of reduction at room temperature. During this process, the hydrogels turned brown in colour, indicating the formation of silver nanoparticles. The sample was then immersed in distilled water for $48 \mathrm{~h}$ to remove unreacted reagents. According to the concentration of the employed silver nitrate solution, the nanocomposite hydrogels are denoted as PU/PVA/Ag-0.5, PU/PVA/Ag-1, PU/PVA/Ag-5 and $\mathrm{PU} / \mathrm{PVA} / \mathrm{Ag}-10$. The synthetic routes of PU/PVA/Ag are illustrated in Scheme 1.

\section{Biocompatibility test}

The to-be-tested PU/PVA and PU/PVA/Ag hydrogels with a size of $5 \mathrm{~mm} \times 5 \mathrm{~mm} \times 1 \mathrm{~mm}$ were sterilized in $75 \%$ ethanol for $30 \mathrm{~min}$ and irradiated with ultraviolet (UV) radiation for $12 \mathrm{~h}$ and used for cell cultures. L929 fibroblast cells (obtained from the American Type Culture Collection, Rockville, MD, USA) were employed to evaluate the biocompatibility of PU/PVA and PU/ PVA/Ag hydrogels. The L929 cells were cultured in Dulbecco's modified Eagle's medium supplemented with fetal bovine serum, penicillin-streptomycin (100 UI $\mathrm{mL}^{-1}$ ) and D-glucose ( $4.5 \mathrm{~g} \mathrm{~L} \mathrm{~L}^{-1}$ ) under $5 \%$ aseptic $\mathrm{CO}_{2}$ and at $37^{\circ} \mathrm{C}$. The culture medium was refreshed every two days. Single cell suspensions were prepared by trypsin digestion when the cells were filled with the medium. Then, PU/PVA and PU/PVA/Ag hydrogels were placed into a 24-well plate and seeded with $10^{4}$ cells $\mathrm{mL}^{-1}$ concentration on each well. Within a given period $(1,2$ and 4 days), $100 \mu \mathrm{L}$ of MTT solution was injected into each well, and then the cells were cultured for another $4 \mathrm{~h}$. After the completion of the culture, the blue formazan reaction product was dissolved by adding $750 \mu \mathrm{L}$ of DMSO, while the upper solvent was discarded. The resultant mixed solution was transferred into a 96-well plate, and its absorbance was measured using a microplate reader (Bio-Rad iMark) three times, and the average absorbance value was calculated.

\section{Antibacterial activity evaluation}

The colony counts method was applied to detect the antibacterial activity of PU/PVA and PU/PVA/Ag nanocomposite hydrogels. The bacterial suspension liquid of common pathogens during the logarithmic phase (Gram-negative Escherichia coli denoted as E. coli and Gram-positive Staphylococcus aureus denoted as $S$. aureus) with a concentration of $1.0 \times 10^{6} \mathrm{CFU} \mathrm{\textrm {mL } ^ { - 1 }}$ was prepared. After centrifugation at $8000 \mathrm{rpm}$ for $10 \mathrm{~min}$, the

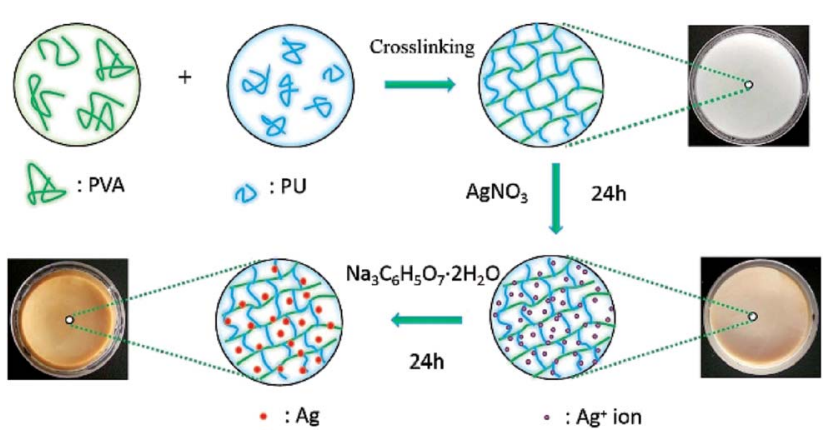

Scheme 1 Synthesis of PU/PVA/Ag hydrogel networks. 
suspension liquid was split into the removed supernatant and precipitate, which was diluted to the original concentration with sterilized physiological saline. The samples of the control and experimental groups were put into Eppendorf tubes with $1 \mathrm{~mL}$ bacterial suspension $\left(10^{6} \mathrm{CFU} \mathrm{mL}^{-1}\right)$. Then, the Eppendorf tubes were incubated in a shaker at $250 \mathrm{rpm}$ for $24 \mathrm{~h}$ at $37{ }^{\circ} \mathrm{C}$. The suspension interacting with the samples was diluted to a concentration of $10^{6} \mathrm{CFU} \mathrm{mL} \mathrm{m}^{-1}$ using a tenfold gradient dilution method. Then, $100 \mu \mathrm{L}$ of the suspension was taken out and coated on the culture medium. After that, the culture medium was incubated for $24 \mathrm{~h}$ at $37^{\circ} \mathrm{C}$ to form colony units, and then the number of colonies on each plate was counted. The samples of each experimental group and the control group were tested three times in parallel to ensure the accuracy of the experiment. The antibacterial rate formula is as follows:

$$
R(\%)=\frac{B-C}{B} \times 100 \%
$$

where $R(\%)$ is the antibacterial rate, where the value takes four significant digits, $B$ is the number of colonies recovered in the control panel after $24 \mathrm{~h}$, and $C$ is the number of colonies recovered in the experimental group panel after $24 \mathrm{~h}$.

\section{Characterizations}

The chemical composition and structural information were determined by Fourier transform infrared spectroscopy (FTIR; Nicolet iS10; 4000-400 $\mathrm{cm}^{-1}$; resolution of $1 \mathrm{~cm}^{-1}$ ) and X-ray photoelectron spectroscopy (XPS; PHI-5702; equipped with a monochromated $\mathrm{Al}-\mathrm{K} \alpha$ radiation source with a chamber pressure of $3 \times 10^{-8}$ Torr). The porous morphology was examined using field-emission scanning electron microscopy (SEM; JSM-6701F) at an accelerating voltage of $5 \mathrm{kV}$. The mechanical properties of the hydrogels (length $70 \mathrm{~mm} \times$ width $10 \mathrm{~mm} \times$ thickness $1 \mathrm{~mm}$ ) were tested on a microcomputercontrolled electronic universal testing machine (WDT-5, Tianshui Hongshan Testing Machine Co., Ltd, China). The stretching speed was kept at $30 \mathrm{~mm} \mathrm{~min}^{-1}$. The friction testing of a stainless ball (10 $\mathrm{mm}$ in diameter) against the PU/PVA/Ag hydrogels was performed on a ball-on-disc tribometer (CSM Instruments, Switzerland), with a reciprocating sliding frequency of $2 \mathrm{~Hz}$ and an applied normal load of $1 \mathrm{~N}$.

\section{Result and discussion}

Fig. 1 presents the FTIR spectra of PVA, PU and PU/PVA. For PU, characteristic peaks at $3345 \mathrm{~cm}^{-1}(\mathrm{~N}-\mathrm{H}$ stretching), 2865$2950 \mathrm{~cm}^{-1}$ (C-H stretching), $1725 \mathrm{~cm}^{-1}$ (free $\mathrm{C}=\mathrm{O}$ stretching), $1625 \mathrm{~cm}^{-1}$ (hydrogen-bonded $\mathrm{C}=\mathrm{O}$ stretching), $1471 \mathrm{~cm}^{-1}$ and $1367 \mathrm{~cm}^{-1}$ (C-H bending), and $1244 \mathrm{~cm}^{-1}$ and $1182 \mathrm{~cm}^{-1}$ (C-O-C stretching) can be observed..$^{25-27}$ From the FTIR spectrum of PVA, peaks at $3410 \mathrm{~cm}^{-1}$ (O-H stretching), 2897$2954 \mathrm{~cm}^{-1}$ (C-H stretching), $1738 \mathrm{~cm}^{-1}$ (residual acetate $\mathrm{CH}_{3} \mathrm{CO}$ ), $1248-1452 \mathrm{~cm}^{-1}$ (C-H bending) and 1005-1144 $\mathrm{cm}^{-1}$ (C-O-C stretching) were found. ${ }^{28,29}$ For the sample of PU/PVA, the broad peak around $3334 \mathrm{~cm}^{-1}$ should be ascribed to $\mathrm{N}-\mathrm{H}$ and $\mathrm{O}-\mathrm{H}$ stretching. The reduced intensity of $-\mathrm{OH}$ compared with PVA should come from the chemical reaction between the
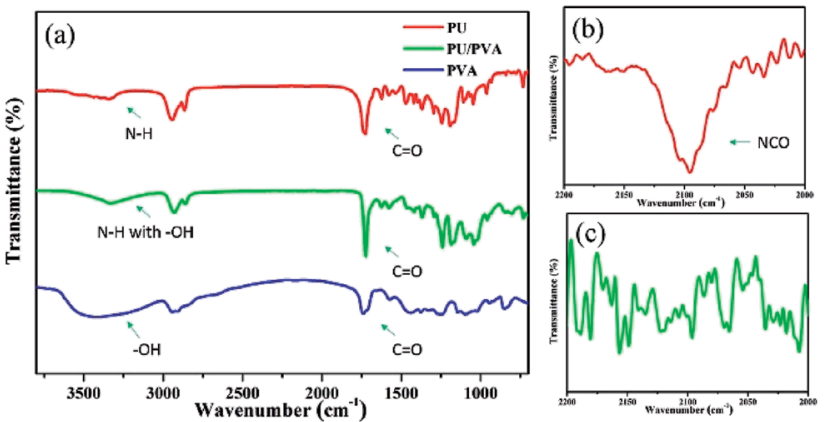

Fig. 1 (a) FTIR spectra of PU, PVA and PU/PVA hydrogels; (b and c) are the magnified FTIR spectra from 2000 to $2200 \mathrm{~cm}^{-1}$ of PU and PU/ PVA, respectively.

isocyanate peak (NCO) group of $\mathrm{PU}$ and $-\mathrm{OH}$ of PVA. To further confirm the above chemical crosslinking, the FTIR spectra of PU and PU/PVA from 2000 to $2200 \mathrm{~cm}^{-1}$ was magnified and compared as shown in Fig. $1 \mathrm{~b}$ and $\mathrm{c}$. It is obviously found that the NCO stretching peak at $c a .2095 \mathrm{~cm}^{-1}$ disappears in PU/ PVA. ${ }^{25}$ Moreover, the peak of hydrogen-bonded $\mathrm{C}=\mathrm{O}$ stretching at $1625 \mathrm{~cm}^{-1}$ becomes weaker for PU/PVA. The mobility of molecular chains was restrained by the reaction between $-\mathrm{OH}$ and -NCO, which may prevent the formation of hydrogenbonded $\mathrm{C}=\mathrm{O}$ in the PU/PVA hydrogel. The abovementioned results suggest that PU and PVA were well crosslinked, and the PU/PVA hydrogel was formed.

Based on the formation of PU/PVA, silver particles were further composited via the solution reduction method at room temperature. The solution reduction method has been widely adopted to prepare silver nanoparticles for various uses. ${ }^{30}$ Fig. 2 shows the XPS Ag 3d spectra of PU/PVA and PU/PVA/Ag series to confirm the existence of silver. With the increase in the Ag concentration, the XPS Ag 3d spectrum becomes gradually remarkable, which involves two peaks at around 373.9 and $367.9 \mathrm{eV}$, corresponding to $\mathrm{Ag} 3 \mathrm{~d}_{3 / 2}$ and $\mathrm{Ag} 3 \mathrm{~d}_{5 / 2}$, respectively. The energy difference is calculated to be $6.0 \mathrm{eV}$, confirming that metallic silver $\left(\mathrm{Ag}^{0}\right)$ has been immobilized in the PU/PVA hydrogels. ${ }^{31}$

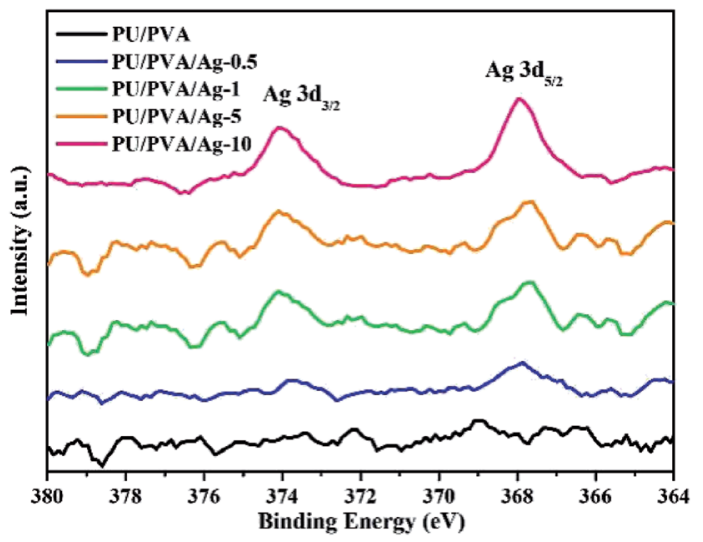

Fig. 2 High-resolution XPS Ag 3d spectra of PU/PVA and the PU/PVA/ Ag composite hydrogels. 
Fig. 3 shows the SEM images of PU/PVA and PU/PVA/Ag to observe their microstructure. It can be found that the hydrogels exhibit a porous structure. Such a structure is advantageous for wound healing because it could allow the supply of oxygen, absorption of the exudate, and maintenance of a moist environment. The pore diameter of PU/PVA is estimated to be $5-10 \mu \mathrm{m}$, which is gradually decreased along with an increase in Ag. The decreased pore diameter of hydrogels after compositing $\mathrm{Ag}$ should be related to the binding of silver nanoparticles with electron-rich $\mathrm{O}$ and $\mathrm{N}$ atoms of the polyurethane chains, which could produce additional cross-linking. ${ }^{32,33}$

The swelling capacity of the hydrogels plays an important role in the wound healing process and other biomedical applications. Better wound dressings should have adequate water absorption capacity for the sake of absorbing wound exudates. ${ }^{34}$ As shown in Fig. 4, the swelling degree of PU/PVA and PU/PVA/ $\mathrm{Ag}$ hydrogels increases with extended soaking time and reaches equilibrium after $6 \mathrm{~h}$ of soaking. The order of the swelling capacity of hydrogels is PU/PVA $>$ PU/PVA/Ag-0.5 $>$ PU/PVA/Ag-1 $>$ PU/PVA/Ag-5 > PU/PVA/Ag-10. The lowered swelling capacity of $\mathrm{PU} / \mathrm{PVA} / \mathrm{Ag}$ hydrogels should be ascribed to the decreased pore diameter of the hydrogels as revealed in Fig. 3. The higher crosslinks limit the infiltration of water in the hydrogels network for swelling. ${ }^{32,33}$ Even so, the PU/PVA/Ag hydrogels show a water absorption capacity of $150-250 \%$.

Since skin is a motorial tissue accompanying various motions, the mechanical behaviour investigation of wound dressings is required to ensure its daily activities. Fig. 5 depicts the stress-strain curves of $\mathrm{PU} / \mathrm{PVA}$ and $\mathrm{PU} / \mathrm{PVA} / \mathrm{Ag}$ nanocomposite hydrogels with different silver loadings. The corresponding summary of Young's modulus, tensile strength and elongation at break are given in Table S1 (ESI†). With the introduction of Ag and further increasing its concentration, the Young's modulus is improved from 0.12 to $0.34 \mathrm{MPa}$, while the tensile strength and elongation at break are significantly improved from 0.05 to $0.52 \mathrm{MPa}$ and from $200 \%$ to $621 \%$, respectively. The enhanced mechanical activities of PU/PVA/Ag hydrogels should also originate from the additional crosslink between Ag and the PU chains. ${ }^{32,33}$ In order to further characterize the mechanical properties of PU/PVA hydrogels, we carried out tensile loading-unloading tests at a constant speed

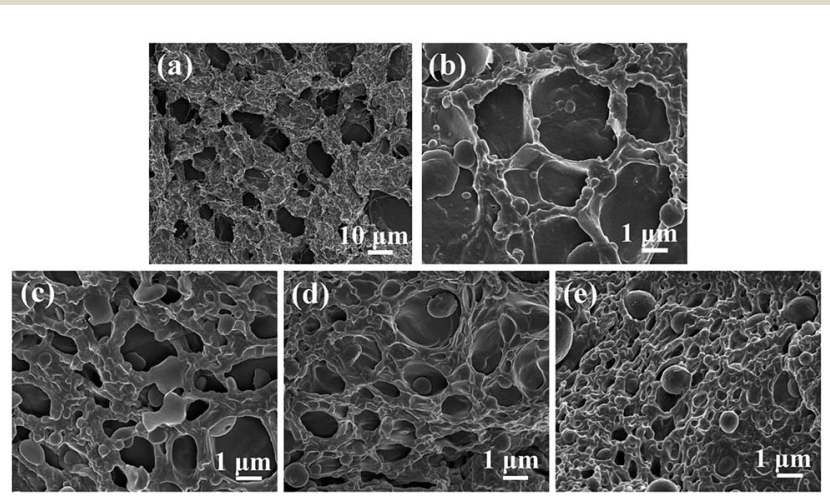

Fig. 3 SEM images of the cross-sections of (a) PU/PVA; (b) PU/PVA/ Ag-0.5; (c) PU/PVA/Ag-1; (d) PU/PVA/Ag-5 and (e) PU/PVA/Ag-10 hydrogels.

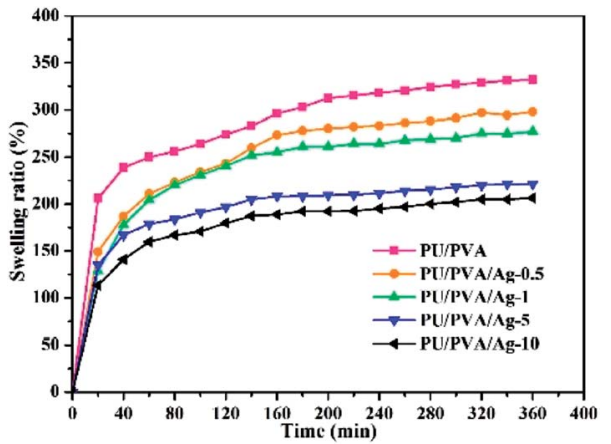

Fig. 4 Swelling ability of PU/PVA and PU/PVA/Ag hydrogels soaked in deionized water for various times.

of $30 \mathrm{~mm} \mathrm{~min}{ }^{-1}$ to evaluate the energy dissipation. Apparent hysteresis loops are observed in the loading-unloading curves of PU/PVA/Ag-10 hydrogel at different strains in Fig. 5b, indicating that the PU/PVA/Ag-10 hydrogel dissipates energy effectively. ${ }^{35}$ It is found that the PU/PVA/Ag-10 hydrogel could dissipate energy of about $282.4 \mathrm{~kJ} \mathrm{~m}^{-3}$ at a strain of $400 \%$. Fig. 5c shows the loading-unloading tests of PU/PVA hydrogel and PU/PVA/Ag nanocomposite hydrogels at the same strain $(200 \%)$. Although the apparent hysteresis loops are observed in all hydrogels, the hysteresis loop of PU/PVA/Ag-10 hydrogel is the largest. The corresponding dissipation energy of PU/PVA/ Ag-10, PU/PVA/Ag-5, PU/PVA/Ag-1, PU/PVA/Ag-0.5, and PU/PVA hydrogels are $39.87,33.97,30.13,30.01$, and $18.36 \mathrm{~kJ} \mathrm{~m}^{-3}$, respectively. It indicates that the introduction of Ag could make the PU/PVA/Ag hydrogels dissipate energy more efficiently than the PU/PVA hydrogel, causing excellent mechanical properties such as extensibility and toughness. ${ }^{36}$ It is noteworthy that the energy dissipation is irreversible during the stretching process
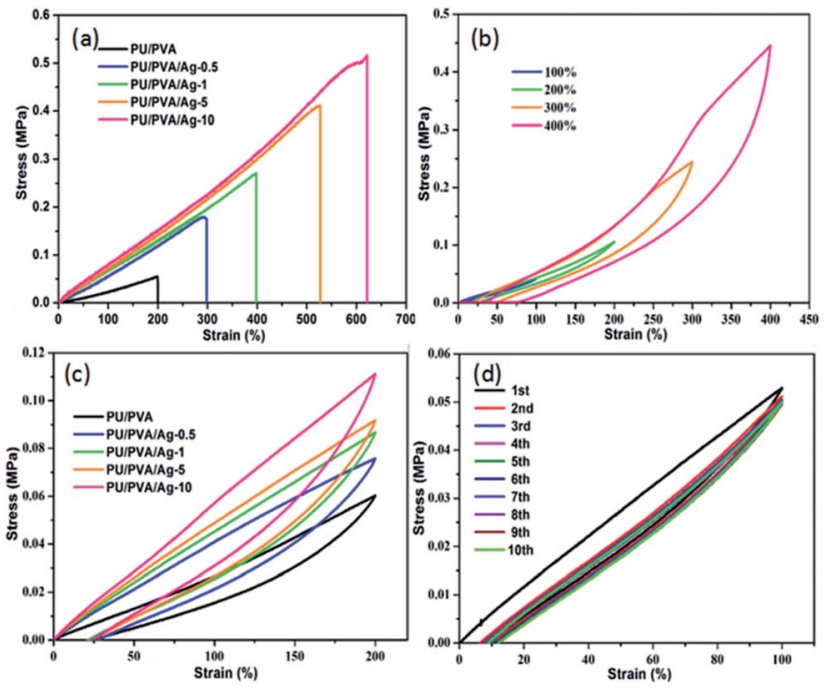

Fig. 5 (a) Tensile stress-strain curves; (b) tensile loading-unloading tests of PU/PVA/Ag-10 hydrogel under different strains (100\%, 200\%, $300 \%, 400 \%$; (c) tensile loading-unloading tests of PU/PVA hydrogel and PU/PVA/Ag nanocomposite hydrogels under $200 \%$ strain; (d) ten cyclic tensile loading-unloading tests of PU/PVA/Ag-10 hydrogel. 
as seen in Fig. 5d. It is found that the first cycle shows a significant hysteresis loop, while the hysteresis becomes negligible and overlaps during the next nine loading-unloading processes. This indicates that the network structure of hydrogels has an irreversible damage. ${ }^{37}$

Fig. 6 shows the friction coefficient of PU/PVA and PU/PVA/Ag nanocomposite hydrogels. For the PU/PVA, PU/PVA/Ag-0.5, PU/PVA/Ag-1 and PU/PVA/Ag-5 hydrogels, the friction coefficient decreased along with an increase in the Ag concentration. The nanoscale polishing effect should be responsible for the reduction of the friction coefficient. With an appropriate size, hardness and volume content, nanoparticles could polish the counterpart surface to a very fine scale, which could reduce the related shear stress and thus decrease the friction coefficient. ${ }^{38}$ In addition to the nanoscale polishing effect, the rolling effect of nanoparticles should also exist, which has been reported to reduce both the frictional coefficient and the wear rate. ${ }^{39}$ When the Ag concentration is further increased to form PU/PVA/Ag-10, its friction coefficient exhibits an increasing trend, which should be aroused by the enhanced aggregation degree of $\mathrm{Ag}$ nanoparticles in the composite hydrogels. The tribological tests suggest that the $\mathrm{PU} / \mathrm{PVA} / \mathrm{Ag}$ nanocomposite hydrogels possess a moderate friction coefficient from 0.2 to 0.35 , which is close to the friction coefficient of human skin as described in literature. ${ }^{40}$

In addition to the porous structure and good mechanical and tribological properties, biocompatibility is another criterion to estimate the feasibility of wound dressing. Biocompatibility is often assessed using the MTT assay that is commonly used to analyse the possible harmful effects induced in cells by materials, with which the cellular mitochondria activity upon exposure to materials is quantified. ${ }^{\mathbf{4 1 , 4 2}}$ For this purpose, PU/PVA/Ag nanocomposite hydrogels were selected as the experimental groups, and the PU/PVA hydrogel was selected as the control group, where L929 cells were seeded directly onto the surface of the samples. As shown in Fig. 7, the cell viability of the PU/PVA/ Ag-0.5, PU/PVA/Ag-1 and PU/PVA/Ag-5 nanocomposite hydrogels tends to be close to or higher than that of the control group. The PU/PVA/Ag-10 nanocomposite hydrogels exhibit lower cell viability in 1 day and 2 day incubations, but show higher cell viability than the control group in 4 day incubations. In brief, the cell viability of the PU/PVA/Ag-10 nanocomposite hydrogels

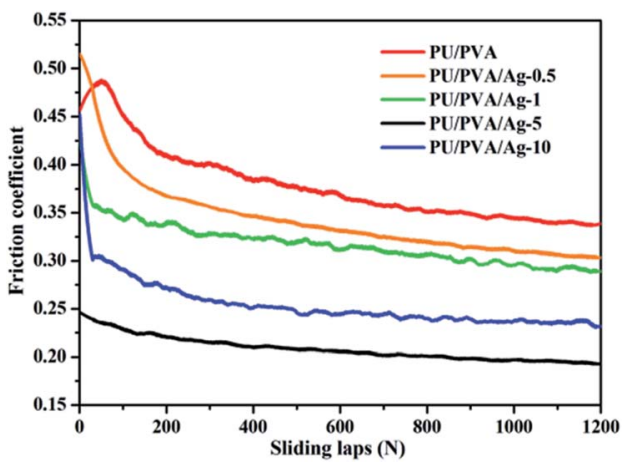

Fig. 6 The friction coefficients of PU/PVA hydrogels and PU/PVA/Ag nanocomposite hydrogels.

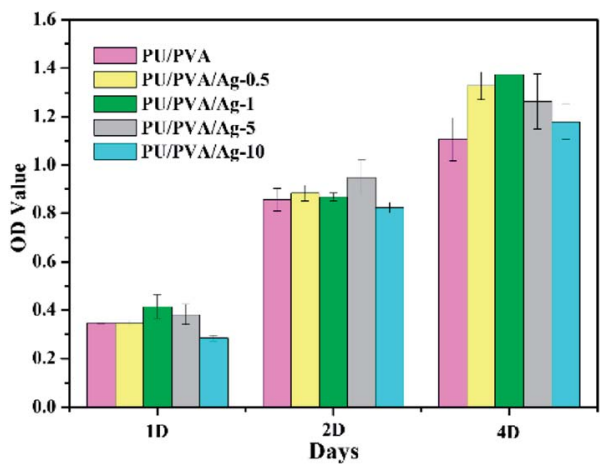

Fig. 7 Cellular proliferation analysis of PU/PVA and PU/PVA/Ag hydrogels.

Table 1 Bacterial reduction percent of the samples

\begin{tabular}{lll}
\hline Sample & E. coli $(\%)$ & S. aureus $(\%)$ \\
\hline PU/PVA & 0 & 0 \\
PU/PVA/Ag-0.5 & $59.84 \pm 1.79$ & $46.20 \pm 3.78$ \\
PU/PVA/Ag-1 & $85.74 \pm 0.7$ & $60.68 \pm 6.90$ \\
PU/PVA/Ag-5 & $93.60 \pm 1.18$ & $81.93 \pm 2.13$ \\
PU/PVA/Ag-10 & $97.07 \pm 0.44$ & $94.61 \pm 0.54$
\end{tabular}

is lower than that of the other experimental groups, which is probably attributed to the aggregation of $\mathrm{Ag}$ nanoparticles, causing slight harmful effects to cell viability. While the cell viability of the remaining experimental groups is better, it indicates that PU/PVA/Ag-0.5, PU/PVA/Ag-1 and PU/PVA/Ag-5 have no cytotoxicity and good biocompatibility.

Bacterial infection is a vital factor for wound healing, and thus we investigate the antimicrobial activities of the asprepared hydrogels to estimate their promising applications as wound dressings. Fig. S1 and S2 (ESI $\dagger$ ) show the antibacterial activities of PU/PVA, PU/PVA/Ag-0.5, PU/PVA/Ag-1, PU/PVA/Ag-5 and PU/PVA/Ag-10 hydrogels determined by the colony counts method. The results are summarized in Table 1 . It is found that the PU/PVA hydrogels show no antibacterial activity, and the incorporation of silver nanoparticles in the hydrogels could induce a dose-dependent antibacterial behavior. This should be attributed to the fact that silver nanoparticles could interact with sulfur-containing proteins as well as phosphoruscontaining compounds like DNA in cells, attacking the respiratory chain with cell division leading to cell death. ${ }^{\mathbf{4 3}}$ For the PU/ PVA/Ag-5 and PU/PVA/Ag-10 hydrogels, their disinfection rates are both higher than $90 \%$. Based on the comprehensive investigation of the swelling ability, mechanical and tribological performance, biocompatibility, and antibacterial behaviour, PU/PVA/Ag-5 nanocomposite hydrogel is considered to be the optimum sample, showing promising applications as an artificial skin for wound healing.

\section{Conclusions}

A series of PU/PVA and PU/PVA/Ag hydrogels was prepared by the chemical cross-linking and in situ synthesis techniques. PU/ 
PVA hydrogels show a uniform porous structure, good swelling ability and biocompatibility. The composition of silver nanoparticles in PU/PVA hydrogels could still maintain the abovementioned properties over a moderate range, while further improving their mechanical and tribological performance, i.e., Young's modulus, tensile strength, elongation at break and friction coefficient. In addition, the PU/PVA/Ag composite hydrogels show excellent antibacterial properties, suggesting that they could be employed for wound healing applications.

\section{Conflicts of interest}

There are no conflicts to declare.

\section{Acknowledgements}

This work is supported by the CAS Pioneer Hundred Talents Program, the National Natural Science Foundation of China (81571829), and the Basic Research Projects of Higher Education Institutions in Gansu Province.

\section{Notes and references}

1 M. Morales-Hurtado, X. Zeng, P. Gonzalez-Rodriguez, J. E. Ten Elshof and E. van der Heide, J. Mech. Behav. Biomed. Mater., 2015, 46, 305-317.

2 I. Yannas and J. F. Burke, J. Biomed. Mater. Res., Part A, 1980, 14, 65-81.

3 C. K. Svensson, Drug Metab. Dispos., 2009, 37, 247-253.

4 J.-P. Chen, G.-Y. Chang and J.-K. Chen, Colloids Surf., A, 2008, 313, 183-188.

5 E. Kamoun, E. Kenawy and X. Chen, J. Adv. Res., 2017, 8, 217233.

6 G. D. Nicodemus and S. J. Bryant, Tissue Eng., Part B, 2008, 14, 149-165.

7 K. Raemdonck, J. Demeester and S. De Smedt, Soft Matter, 2009, 5, 707-715.

8 L. Engel, O. Berkh, K. Adesanya, J. Shklovsky, E. Vanderleyden, P. Dubruel, Y. Shacham-Diamand and S. Krylov, Sens. Actuators, B, 2014, 191, 650-658.

9 A. Wada, S.-i. Tamaru, M. Ikeda and I. Hamachi, J. Am. Chem. Soc., 2009, 131, 5321-5330.

10 L. Wu, G. Chen and Z. Li, Small, 2017, 13, 1604070.

11 L. Wu, C. Gao, Z. Li and G. Chen, J. Mater. Chem. C, 2017, 5, 5207-5213.

12 Z. Gong, G. Zhang, X. Zeng, J. Li, G. Li, W. Huang, R. Sun and C. Wong, ACS Appl. Mater. Interfaces, 2016, 8, 24030-24037.

13 J. P. Gong, Y. Katsuyama, T. Kurokawa and Y. Osada, Adv. Mater., 2003, 15, 1155-1158.

14 Y. Okumura and K. Ito, Adv. Mater., 2001, 13, 485-487.

15 Z. Hu and G. Chen, Adv. Mater., 2014, 26, 5950-5956.

16 L. Wu, Z. Hu, G. Chen and Z. Li, Soft matter, 2015, 11, 90389044.

17 M. Kokabi, M. Sirousazar and Z. M. Hassan, Eur. Polym. J., 2007, 43, 773-781.

18 Z. Fan, B. Liu, J. Wang, S. Zhang, Q. Lin, P. Gong, L. Ma and S. Yang, Adv. Funct. Mater., 2014, 24, 3933-3943.
19 D. Staneva, D. Atanasova, E. Vasileva-Tonkova, V. Lukanova and I. Grabchev, Appl. Surf. Sci., 2015, 345, 72-80.

20 K.-D. Vorlop, A. Muscat and J. Beyersdorf, Biotechnol. Tech., 1992, 6, 483-488.

21 L. Rao, H. Zhou, T. Li, C. Li and Y. Y. Duan, Acta Biomater., 2012, 8, 2233-2242.

22 S. Bonakdar, S. H. Emami, M. A. Shokrgozar, A. Farhadi, S. A. H. Ahmadi and A. Amanzadeh, Mater. Sci. Eng., C, 2010, 30, 636-643.

23 L. Zhou, D. Liang, X. He, J. Li, H. Tan, Q. Fu and Q. Gu, Biomaterials, 2012, 33, 2734-2745.

24 H. J. Yoo and H. D. Kim, J. Biomed. Mater. Res., Part B, 2008, 85, 326-333.

25 G. Trovati, E. A. Sanches, S. C. Neto, Y. P. Mascarenhas and G. O. Chierice, J. Appl. Polym. Sci., 2010, 115, 263-268.

26 L. Jiao, H. Xiao, Q. Wang and J. Sun, Polym. Degrad. Stab., 2013, 98, 2687-2696.

27 G. Chen, Y. Ma, X. Zheng, G. Xu, J. Liu, J. Fan, D. Shen and Z. Qi, J. Polym. Sci., Part B: Polym. Phys., 2007, 45, 654660.

28 G. Andrade, E. Barbosa-Stancioli, A. P. Mansur, W. Vasconcelos and H. Mansur, Biomed. Mater., 2006, 1, 221.

29 N. V. Bhat, M. M. Nate, M. B. Kurup, V. A. Bambole and S. Sabharwal, Nucl. Instrum. Methods Phys. Res., Sect. B, 2005, 237, 585-592.

30 A. R. Shahverdi, A. Fakhimi, H. R. Shahverdi and S. Minaian, Nanomedicine, 2007, 3, 168-171.

31 H. Kong and J. Jang, Chem. Commun., 2006, 3010-3012.

32 K. Vimala, Y. M. Mohan, K. S. Sivudu, K. Varaprasad, S. Ravindra, N. N. Reddy, Y. Padma, B. Sreedhar and K. MohanaRaju, Colloids Surf., B, 2010, 76, 248-258.

33 K. Varaprasad, K. Vimala, S. Ravindra, N. N. Reddy, G. V. S. Reddy and K. M. Raju, J. Mater. Sci., 2011, 22, 1863-1872.

34 T. Wang, X.-K. Zhu, X.-T. Xue and D.-Y. Wu, Carbohydr. Polym., 2012, 88, 75-83.

35 P. Lin, S. Ma, X. Wang and F. Zhou, Adv. Mater., 2015, 27, 2054-2059.

36 Z. Gong, G. Zhang, X. Zeng, J. Li, G. Li, W. Huang, R. Sun and C. Wong, ACS Appl. Mater. Interfaces, 2016, 8, 24030-24037.

37 Y. Liu, W. Zhou, Q. Zhou, K. Peng, A. Yasin and H. Yang, RSC Adv., 2017, 7, 29489-29495.

38 L. Chang, Z. Zhang, C. Breidt and K. Friedrich, Wear, 2005, 258, 141-148.

39 L. Kahlman and I. M. Hutchings, Tribol. Trans., 1999, 42, 842-850.

40 A. B. Cua, K. P. Wilhelm and H. Maibach, Br. J. Dermatol., 1990, 123, 473-479.

41 F. M. Young, W. Phungtamdet and B. J. Sanderson, Toxicol. In Vitro, 2005, 19, 1051-1059.

42 Z. Fan, J. Wang, Z. Wang, H. Ran, Y. Li, L. Niu, P. Gong, B. Liu and S. Yang, Carbon, 2014, 66, 407-416.

43 J. S. Kim, E. Kuk, K. N. Yu, J.-H. Kim, S. J. Park, H. J. Lee, S. H. Kim, Y. K. Park, Y. H. Park and C.-Y. Hwang, Nanomedicine, 2007, 3, 95-101. 